Review Article

\title{
Correlation between Soluble $\alpha$-Klotho and Renal Function in Patients with Chronic Kidney Disease: A Review and Meta-Analysis
}

\author{
Qinglian Wang $\mathbb{D}^{1},{ }^{1}$ Wenyan Su, ${ }^{1}$ Zhenwei Shen, $^{2}$ and Rong Wang ${ }^{1}{ }^{1}$ \\ ${ }^{1}$ Department of Nephrology, Shandong Provincial Hospital Affiliated to Shandong University, Jinan, China \\ ${ }^{2}$ Qilu Pharmaceutical Co., Ltd., Jinan, China \\ Correspondence should be addressed to Rong Wang; wrjlsd@126.com
}

Received 15 December 2017; Revised 29 May 2018; Accepted 11 July 2018; Published 12 August 2018

Academic Editor: Peyman Björklund

Copyright (C) 2018 Qinglian Wang et al. This is an open access article distributed under the Creative Commons Attribution License, which permits unrestricted use, distribution, and reproduction in any medium, provided the original work is properly cited.

Objective. Over decades, numerous inconsistent studies are reported on the relationship between soluble $\alpha$-Klotho and renal function in patients with chronic kidney disease (CKD). This study aims to perform a meta-analysis to figure out the correlations between soluble $\alpha$-Klotho and renal function in patients with CKD. Materials and Methods. We searched medical and scientific literature databases, PubMed and EMBASE (from the inception to October 2017), for publications that reported studies on associations between soluble $\alpha$-Klotho and renal function in patients with CKD. Only publications in English were extracted. Summary correlation coefficient (r) values were extracted from each study, and 95\% confidence intervals (CIs) were calculated. Publication bias was tested, and sensitivity and subgroup analyses were performed to investigate potential heterogeneity. Results. Of 611 studies, 9 publications with 1457 patients were included into the analysis. The following data were extracted from the literature: first author, year of publication, research region, research index, sample size, average age and Pearson or Spearman correlation coefficient, study design, the $\alpha$ Klotho/FGF23 assays utilized, full length, or the C-terminal fragment of FGF23. The pooled $\mathrm{r}$ between $\alpha$-Klotho and estimated glomerular filtration rate (eGFR), FGF-23 were 0.35 (95\%CI, 0.23 0.46, and $\mathrm{P}<0.05)$, -0.10 (95\%CI, -0.19 0.01 , and $\mathrm{P}<0.05)$ with remarkable significance, indicating moderate heterogeneity. There was no significant heterogeneity between subgroups in analyses of $\alpha$-Klotho and eGFR stratified by research region, mean age, and eGFR, but heterogeneity exists in analyses of $\alpha$-Klotho and FGF-23 stratified by research region. There was no significant correlation between a-klotho and Ca and PTH and PHOS. There was no evidence of publication bias with Egger's test $(\mathrm{p}=0.360)$ or with Begg's test $(\mathrm{p}=0.902)$ and the distribution of funnel plots was symmetrical in all of our analysis. Conclusions. There exists a significant positive correlation between soluble $\alpha$ Klotho and eGFR in patients with CKD. Also, a significant negative correlation between $\alpha$-Klotho and FGF23 levels is proven. This raises hope to employ $\alpha$ Klotho and FGF23 as early biomarkers of CKD. However, further large prospective follow-up researches are needed to validate this hypothesis and to explore whether maintaining or elevating the Klotho level could improve renal function and complications in CKD patients.

\section{Introduction}

The $\alpha$-Klotho was originally identified as an aging suppressor by Kuro-M et al. in 1977, the year when they found the Klotho-deficient mice displayed phenotypes resembling human premature-aging syndrome with shortened lifespan [1]. The gene encoding a single-pass transmembrane protein is mainly expressed in distal convoluted renal tubules, choroid plexus of the brain [1] and parathyroid [2] and also in pituitary gland [3], inner ear [4], breast epithelial cell [5], and other tissues [1]. As an obligatory coreceptor of fibroblast growth factor 23(FGF23), it forms a complex with fibroblast growth factor receptor (FGFR) $[6,7]$, which can maintain mineral homeostasis by regulating urinary phosphorus reabsorption and inhibiting production of 1,25dihydroxy vitamin $\mathrm{D}$ in kidneys and suppressing synthesis and secretion of parathyroid hormone in parathyroid gland [8]. In addition to its membrane-bond form, there 
TABLE 1: Inclusion and exclusion criteria.

(1) Investigation of the relationship between soluble $\alpha$-klotho (measured in plasma or serum) and renal function associated indexes (eGFR was determined based on CKD-EPI or MDRD) in chronic kidney disease using correlation analyses.

Inclusion criteria

(2) Enrolling adults (mean age $\geq 18$ years old).

(3) Containing complete data information.

(4) Research article published in the peer-reviewed journals.

(1) Examination of the correlation between renal Klotho (mRNA or protein expression) and other related parameters.

(2) Studies that didn't contain correlation coefficients ( $R$ value) between renal function associated indexes in CKD and soluble $\alpha$-klotho protein.

Exclusion criteria

(3) Special populations such as those who suffered from renal replacement therapy or study populations with one specific kidney disease, etc. Diabetic nephrology patients were excluded.

(4) Animal-based experiments, in-vitro experiments, having no insufficient data, having significant overlap in the study populations, all duplicated publications, or enrolling only children

(5) Case reports, posters, reviews and meeting abstracts

is also a soluble form generated by proteolytic release of the transmembrane form or alternative transcript splicing $[9,10]$. As a humoral regulator, it can be detected in plasm, urine, and cerebrospinal fluid. And it has pleiotropic biologic effect including inhibiting the signal transduction pathway of insulin/insulin-like growth factor 1 and Wnt, reducing oxidative stress as well as regulating nitric oxide synthesis [11].

CKD is considered to be an increasing public health issue with low awareness rate and its prevalence rate is about 8 $16 \%$ around the world [12]. The leading cause of morbidity and mortality in patients with CKD is cardiovascular diseases (CVD) which cause great financial burden [13]. In recent years, as kidney function declines, these risk factors such as abnormal calcium-phosphorus metabolism, oxidative stress, and anemia have become conspicuous and ulteriorly increase cardiovascular diseases risks [14]. As these abnormal indexes which could be detected at early stages of CKD predict CKD progression and adverse outcomes, they could be applied as early biomarkers. Hence, identification of CKD at early stages is critical to monitor disease progression and reduce cardiovascular collapses.

In recent years, emerging evidence suggests that the serum soluble $\alpha$-Klotho could serve as an early biomarker for CKD. It has been reported that serum level of soluble $\alpha$ Klotho decreased in the early stage of CKD and there was a significant inverse correlation between soluble $\alpha$-Klotho level and kidney function decline [14-16]. However, some studies have yield contradictory results that soluble Klotho levels were not pertinent to renal function [17-19]. Thus, we conducted a meta-analysis by summarizing the available evidence in order to overall assess the diagnostic value of serum soluble Klotho level for CKD.

\section{Materials and Methods}

2.1. Literature Search. Two researchers independently searched the PubMed (MEDLINE included) and EMBASE library databases from inception to October 2017 for related published studies. Our research was limited to literatures written in English. Index terms we used to search the indicated databases were ((Chronic Kidney Diseases) OR (Chronic Renal Diseases) OR (Chronic Kidney Insufficiency) OR (Chronic Renal Insufficiency) OR (CKD) OR (Chronic nephropathy) OR (Chronic kidney failure) OR (Chronic Renal failure)) AND ((Klotho protein) OR (KL protein) OR (alpha-Klotho protein) OR $(\alpha \mathrm{KL}))$. Secondary references included in these literatures were also recruited.

2.2. Study Identification and Selection. First papers without detailed data and duplicates in terms of CKD and Klotho protein were excluded. Thereafter 611 publications were involved into further analysis. Two independent reviewers evaluated the potentially relevant articles on the basis of the inclusion and exclusion criteria as shown in Table 1.

If there was discordance among the 2 independent researchers for one study, its eligibility was decided by the 3rd investigator. Overall 504 publications were excluded by looking through title and abstract. Then 86 publications were excluded by reading the full articles; therefore 21 publications were reserved. Further by reading thoroughly and discussing together, 12 publications were excluded. Finally 9 publications with 1457 patients were comprised. 9 patients' samples were included. Detailed information about flowchart of the study selection process was shown in Figure 1.

2.3. Quality Assessment. For quality assessment of each included study, we considered items in the Strengthening the Reporting of Observational Studies in Epidemiology (STROBE) guidelines [20]. We focused particularly on the following main characteristics of the study, including the study design and participants' characteristics in the statistical analysis. As there is no consensus on the tools to evaluate the quality of observational cross-sectional studies, this article did not give a comprehensive score. Moreover, a simple application of such scores was discouraged in previous studies [21-23].

2.4. Data Extraction. The data were extracted from the included literatures by two investigators (Qinglian Wang and Wenyan $\mathrm{Su}$ ) independently, and the extracted contents 


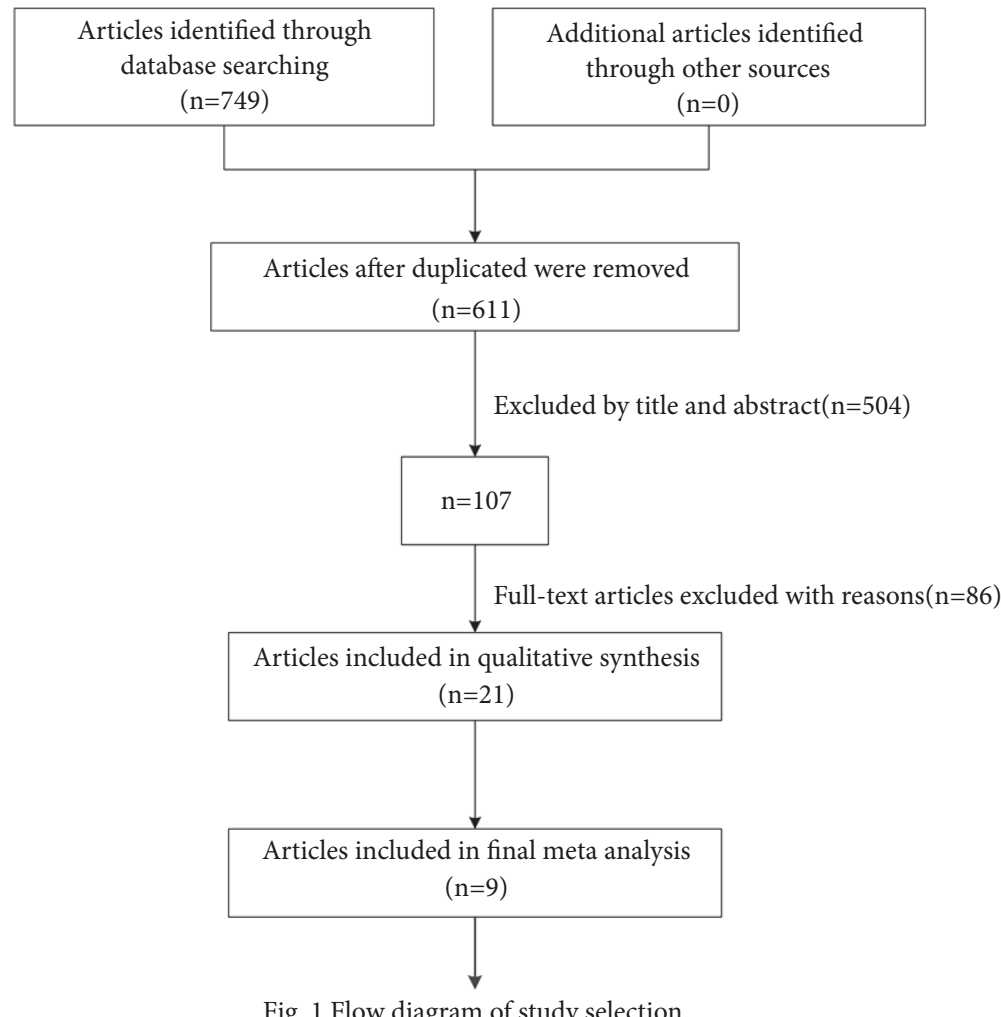

FIGURE 1: Flowchart of the study selection process.

included the following: first author, year of publication, research region, research index, sample size, average age and Pearson or Spearman correlation coefficient, study design, assay utilization about FGF23 and $\alpha$-Klotho, and intact/Cterminal fragment of FGF-23 measured. Spearman and Pearson correlation coefficients were all recorded in different articles. In order to utilize data better, we converted the published Pearson correlation coefficients into Spearman correlation coefficients as the latter was not affected by logarithmic transformation [24]. Furthermore, since the sampling of Spearman correlation coefficient is not normally distributed and the confidence interval (CI) was calculated depending on the value of correlation coefficient. We converted each correlation coefficient to an approximately normally distributed $\mathrm{z}$ value by Fisher's transformation and then calculated the standard error (SE) of $z$. The $z$ value was then converted by inverse Fisher's transformation to obtain correlation coefficient and CI. A third reviewer joined to assess the terms and vote for a decision when disagreement occurred.

2.5. Meta-Analysis. After Fisher's $\mathrm{z} \sim \mathrm{r}$ transformation, random effects meta-analysis was chosen to combine the data [25]. Heterogeneity was obtained by calculating the $Q$ value and inconsistency index (I2) [26]. $\mathrm{P}<0.05$ or $\mathrm{I}^{2}>50 \%$ indicated the existence of heterogeneity. Sensitivity analysis was performed when notable heterogeneity existed to further explore the source of heterogeneity. Studies were stratified by following grouping methods: (1) patients age $(<60 \mathrm{y}$ or $\geq 60 \mathrm{y}),(2)$ research region (Asia or Europe), and (3) eGFR $\left(\geq 45 \mathrm{ml} /\left(\mathrm{min} \cdot 1.73 \mathrm{~m}^{2}\right)\right.$ or $\left.<45 \mathrm{ml} /\left(\mathrm{min} \cdot 1.73 \mathrm{~m}^{2}\right)\right)$.

Publication bias was assessed by Begg's and Egger's test and visually assessed by funnel plot.

All the statistical analyses were carried out using Stata Software (Version 13.0 StataCorp, College Station, TX, USA).

\section{Results}

3.1. Study Characteristics. We included 9 associated studies from 9 publications in our systematic review and metaanalysis (Table 2). Of them, 8 publications [14-18, 27-29] reported data on $\alpha$-Klotho and eGFR, with 1136 participants. 7 publication $[14-16,18,19,27,29]$ reported data on $\alpha$-Klotho and FGF23, with 1239 participants. 4 publications [14, 15, 19, 27] reported data on $\alpha$-Klotho and Ca, with 863 participants. 3 publications $[14,15,19]$ reported data on $\alpha$-Klotho and PTH, with 678 participants. 5 publications $[14,15,19,27,29]$ reported data on $\alpha$-Klotho and PHOS, with 887 participants (Table 2).

The 9 publications were published between 2012 and 2014 . 5 publications were from the AISA and 4 from Europe. All of the 9 studies were conducted in both male and female (Table 2).

\subsection{Results of Meta-Analysis}

3.2.1. $\alpha$-Klotho and eGFR. Figure 2 shows the meta-analysis of the correlation between $\alpha$-Klotho and eGFR. All of the 
TABLE 2: Characteristics of included studies.

\begin{tabular}{|c|c|c|c|c|c|c|c|c|c|}
\hline Study & $\begin{array}{c}\text { Publish } \\
\text { year }\end{array}$ & Nation & $\begin{array}{c}\text { Average } \\
\text { age }\end{array}$ & $\mathrm{N}$ & index & $\begin{array}{c}\text { Correlation } \\
\text { coefficient }\end{array}$ & Study design & Assay utilization & $\begin{array}{c}\text { Intact/C-terminal } \\
\text { of FGF-23 }\end{array}$ \\
\hline \multirow{5}{*}{$\begin{array}{l}\text { Masashi } \\
\text { Kitagawa }\end{array}$} & \multirow{5}{*}{2013} & \multirow{5}{*}{ Japan } & \multirow{5}{*}{58} & \multirow{5}{*}{114} & GFR & $0.3913 *$ & \multirow{5}{*}{$\begin{array}{l}\text { Cross- } \\
\text { sectional }\end{array}$} & \multirow{5}{*}{ ELISA(Japan) } & \multirow{5}{*}{ Intact } \\
\hline & & & & & & $-0.272 *$ & & & \\
\hline & & & & & FGF23 & $-0.1751 *$ & & & \\
\hline & & & & & $\mathrm{Ca}$ & $0.1618 *$ & & & \\
\hline & & & & & Phos & $-0.3034 *$ & & & \\
\hline \multirow{4}{*}{$\begin{array}{l}\text { Michishige } \\
\text { Ozeki }\end{array}$} & \multirow{4}{*}{2014} & \multirow{4}{*}{ Japan } & \multirow{4}{*}{68.9} & \multirow{4}{*}{185} & GFR & $0.209 *$ & \multirow{4}{*}{ Retrospective } & \multirow{4}{*}{ ELISA(Japan) } & \multirow{4}{*}{ Intact } \\
\hline & & & & & FGF23 & -0.14 & & & \\
\hline & & & & & $\mathrm{Ca}$ & $0.06 *$ & & & \\
\hline & & & & & Phos & 0.05 & & & \\
\hline \multirow{2}{*}{ Tetsu Akimotol } & \multirow{2}{*}{2012} & \multirow{2}{*}{ Japan } & \multirow{2}{*}{56} & \multirow{2}{*}{131} & GFR & 0.232 & \multirow{2}{*}{$\begin{array}{c}\text { Cross- } \\
\text { sectional }\end{array}$} & \multirow{2}{*}{ ELISA(Japan) } & \multirow{2}{*}{$\mathrm{Nr}$} \\
\hline & & & & & Age & -0.172 & & & \\
\hline \multirow{5}{*}{$\begin{array}{l}\text { Hyoung Rae } \\
\text { Kim }\end{array}$} & \multirow{5}{*}{2013} & \multirow{5}{*}{ Korea } & \multirow{5}{*}{45.7} & \multirow{5}{*}{243} & GFR & 0.502 & & & \\
\hline & & & & & Age & -0.395 & & & \\
\hline & & & & & FGF23 & -0.245 & $\begin{array}{l}\text { Cross- } \\
\text { sectional }\end{array}$ & ELISA(Japan) & Intact \\
\hline & & & & & $\mathrm{Ca}$ & 0.257 & & & \\
\hline & & & & & Phos & -0.169 & & & \\
\hline Valerie Hage & 2014 & France & 467 & 60 & GFR & 0.11 & Cross- & FIJSA(Janan) & $\mathrm{Nr}$ \\
\hline valeme nage & 2014 & rrance & 40.1 & 00 & FGF23 & 0.23 & sectional & LLIsA(fapan) & NI \\
\hline & & & & & FGF23 & $-0.03 *$ & & & \\
\hline Sarah Seiler & 2012 & Germany & 65.5 & 321 & $\mathrm{Ca}$ & $0.01 *$ & Prospective & ELISA(Japan) & C-terminal \\
\hline & & & & & Phos & $-0.06 *$ & & & \\
\hline Ivana Pavik & 2013 & Switzerland & 52.7 & 87 & GFR & 0.64 & $\begin{array}{c}\text { Cross- } \\
\text { sectional }\end{array}$ & ELISA(Japan) & C-terminal \\
\hline & & & & & GFR & 0.441 & & & \\
\hline $\begin{array}{l}\text { Yosniko } \\
\text { Shimamura }\end{array}$ & 2012 & Japan & 63.8 & 292 & Age & -0.345 & Prospective & ELISA(Japan) & Intact \\
\hline & & & & & FGF23 & -0.156 & & & \\
\hline & & & & & GFR & $0.11 *$ & & & \\
\hline Alexandra & 2014 & Denmark & 68 & 24 & Age & $-0.25 *$ & RCT & ELISA(Japan) & Intact \\
\hline Scholze & 2014 & Deminlák & 00 & 24 & FGF23 & $0.09 *$ & NL1 & 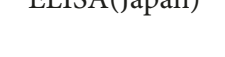 & intact \\
\hline & & & & & Phos & $-0.10 *$ & & & \\
\hline
\end{tabular}

Note. * spearman correlation coefficient, otherwise, Pearson correlation coefficient. Nr representative not reported.

included 8 studies showed a positive correlation between $\alpha$-Klotho and eGFR. There was a significant heterogeneity among the 8 studies $\left(\mathrm{I}^{2}=68.7 \%, \mathrm{P}=0.002\right)$, so we used a random effects model. The pooled Spearman correlation coefficient was 0.35 (95\%CI, 0.23 0.46, and $\mathrm{P}<0.05)$, suggesting that there was a significant positive correlation between $\alpha$-Klotho and eGFR.

There was no evidence of publication bias with Egger's test $(\mathrm{p}=0.360)$ or with Begg's test $(\mathrm{p}=0.902)$. The distribution of funnel plots was symmetrical (Supplementary Materials Figure 1). The result is suggestive of an indistinctive small study bias. In the sensitivity analysis, the pooled RR ranged from 0.32 (95\%CI, 0.19 0.44) to 0.37 (95\%CI, 0.26 0.48) (Supplementary Materials Figure 2). No study had a significant impact on the total combined results.

To explore potential sources of moderate heterogeneity across studies and to examine the impact on final summary estimates, we conducted a series of subgroup analyses according to mean age $(<60 \mathrm{y}$ or $\geq 60 \mathrm{y})$, research region (Asia or Europe), eGFR $\left(\geq 45 \mathrm{ml} /\left(\min \cdot 1.73 \mathrm{~m}^{2}\right)\right.$, or $<45 \mathrm{ml} /$ $\left.\left(\min \cdot 1.73 \mathrm{~m}^{2}\right)\right)$. There was no significant heterogeneity between subgroups in analyses of $\alpha$-Klotho and eGFR stratified by research region, mean age and eGFR ( $P$ for group heterogeneity $>0.05$ ). Positive correlations were apparent in all subgroups, and all of them were statistically significant (Supplementary Materials Table 1).

3.3. $\alpha$-Klotho and FGF-23. Figure 3 ( $\alpha$-Klotho and FGF-23) shows the meta-analysis of the correlation between $\alpha$-Klotho and FGF-23. Among the included 7 studies, 5 found a negative correlation between $\alpha$-Klotho and FGF-23 and 2 found a positive correlation. With a random effects model $\left(\mathrm{I}^{2}=56.6 \%\right.$, $\mathrm{P}=0.032$ ), the pooled Spearman correlation coefficient was 0.10 (95\%CI, $-0.19 \sim-0.01$, and $\mathrm{P}<0.05$ ), suggesting that there 


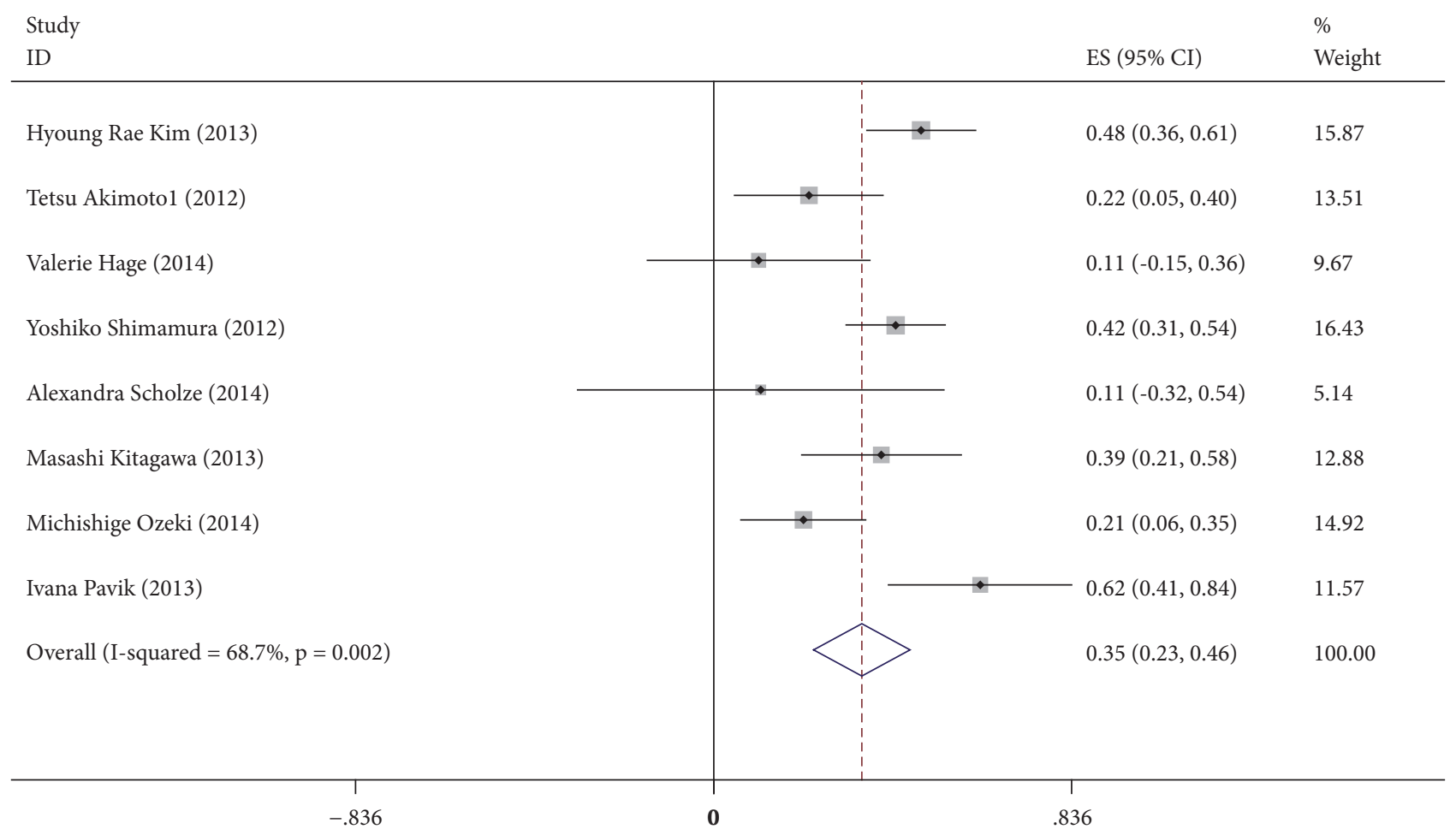

FIGURE 2: Forest plots of the summary correlation coefficient (r) with corresponding 95\% CIs for the correlation between $\alpha$-Klotho and eGFR in patients from all eligible studies.

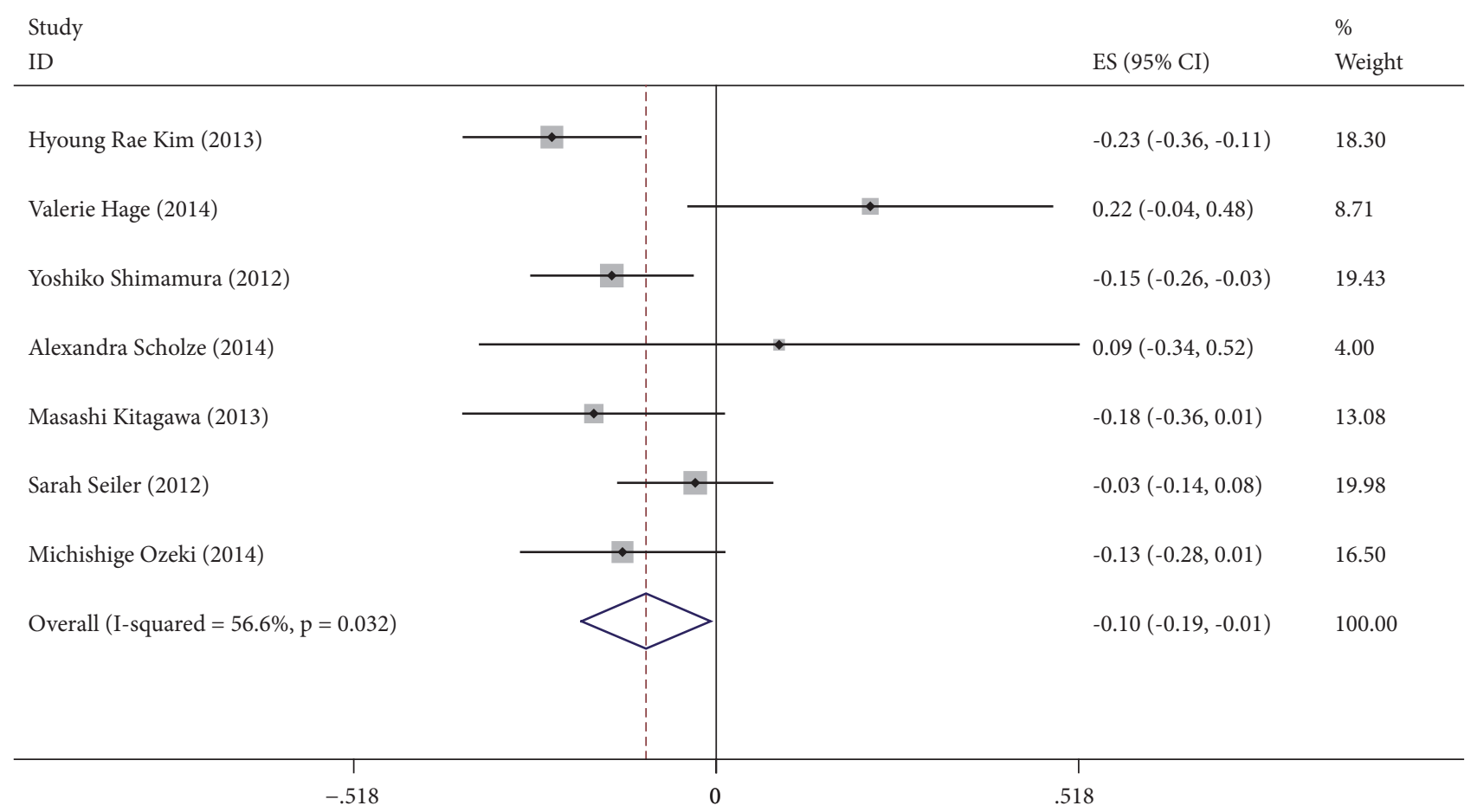

Figure 3: Forest plots of the summary correlation coefficient (r) with corresponding 95\% CIs for the correlation between $\alpha$-Klotho and FGF-23 in patients from all eligible studies. 


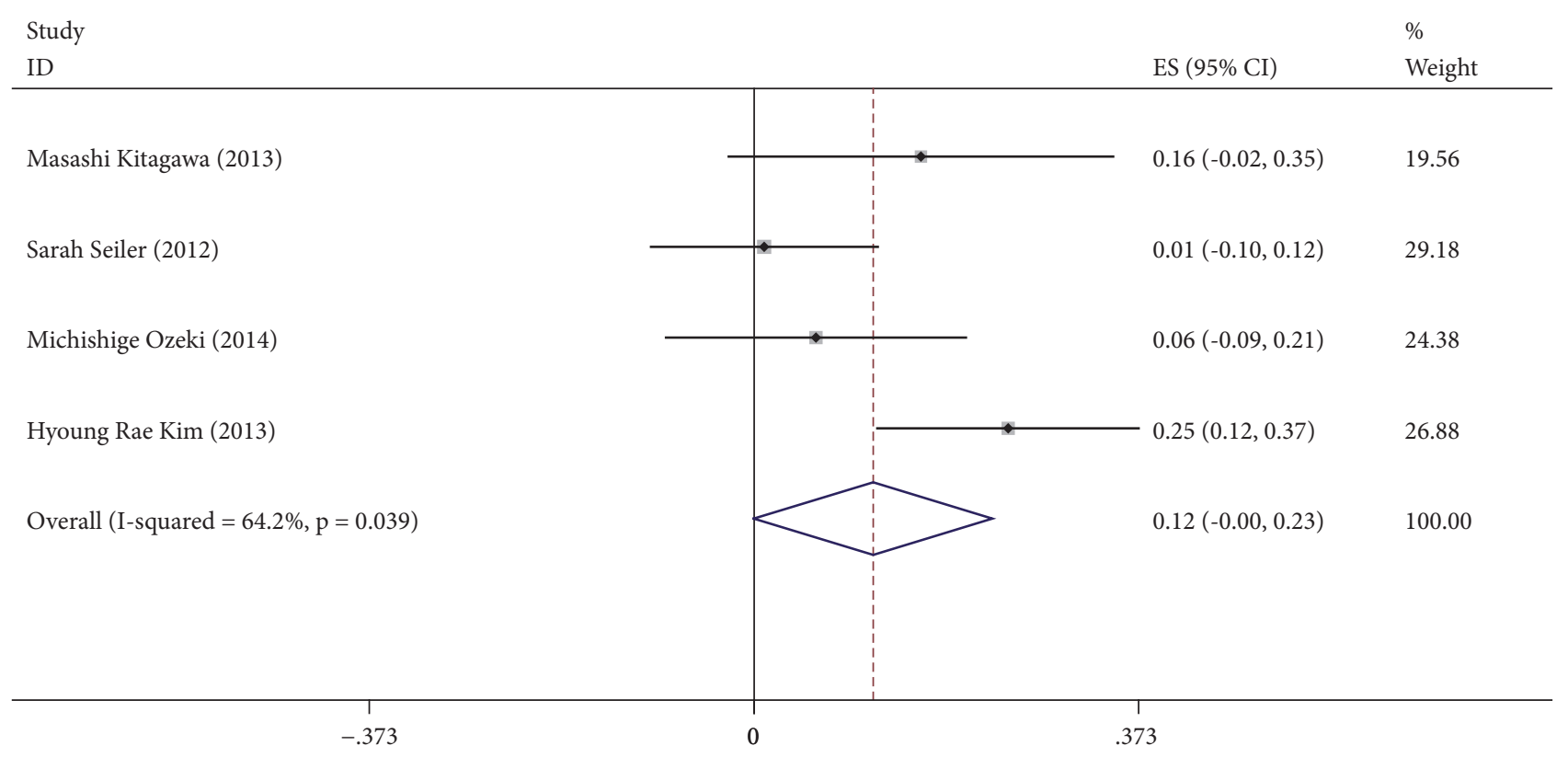

FIGURE 4: Forest plots of the summary correlation coefficient (r) with corresponding 95\% CIs for the correlation between $\alpha$-Klotho and Ca in patients from all eligible studies.

was a significant negative correlation between $\alpha$-Klotho and FGF-23.

There was no evidence of publication bias with Egger's test $(\mathrm{p}=0.781)$ or with Begg's test $(\mathrm{p}=1.000)$. The distribution of funnel plots was symmetrical (Supplementary Materials Figure 3). The result is suggestive of an indistinctive small study bias. In the sensitivity analysis, the pooled RR ranged from $-0.13(95 \% \mathrm{CI},-0.20 \sim-0.06)$ to $-0.07(95 \% \mathrm{CI},-0.17 \sim-$ 0.02) (Supplementary Materials Figure 4). No study had a significant impact on the total combined results.

To explore potential sources of moderate heterogeneity over studies and to examine the impact on final summary estimates, we conducted subgroup analysis according to mean age $(<60 \mathrm{y}$ or $\geq 60 \mathrm{y})$, research region (Aisa or Europe), and eGFR $\left(\geq 45 \mathrm{ml} / \mathrm{min} \cdot 1.73 \mathrm{~m}^{2}\right.$ or $\left.<45 \mathrm{ml} / \mathrm{min} \cdot 1.73 \mathrm{~m}^{2}\right)$. There was no significant heterogeneity between subgroups in analyses of $\alpha$-Klotho and FGF-23 stratified by mean age and eGFR (P group heterogeneity $>0.05$ ). Research regions might be the sources of heterogeneity ( $\mathrm{P}$ group heterogeneity<0.05) and participants in Asia subgroup showed a significant negative correlation between $\alpha$-Klotho and FGF-23, while participants in Europe subgroup showed no correlation. Negative correlations between $\alpha$-Klotho and FGF-23 were apparent in age and eGFR subgroups, and all of them were statistically significant (Supplementary Materials Table 1).

3.4. $\alpha$-Klotho and $\mathrm{Ca}, \mathrm{PTH}$ and PHOS. Figure 4 ( $\alpha$-Klotho and $\mathrm{Ca}$ ) shows the meta-analysis of the correlation between $\alpha$-Klotho and Ca. All of the included 4 studies found a positive correlation between $\alpha$-Klotho and $\mathrm{Ca}$. With a random effects model $\left(\mathrm{I}^{2}=64.2 \%, \mathrm{P}=0.039\right)$, the pooled Spearman correlation coefficient was 0.12 (95\%CI, $0.00 \sim 0.23, \mathrm{P}>0.05)$, suggesting no significant positive correlation between $\alpha$ Klotho and Ca. Figure 5 ( $\alpha$-Klotho and PTH) shows the metaanalysis of the correlation between $\alpha$-Klotho and PTH. We included 3 studies, 1 found a positive correlation between $\alpha$-Klotho and PTH, and 2 reported a negative correlation. With a random effects model $\left(\mathrm{I}^{2}=85.2 \%, \mathrm{P}=0.001\right)$, the pooled Spearman correlation coefficient was -0.14 (95\%CI, -0.34 $0.07, \mathrm{P}>0.05)$, suggesting no significant correlation between $\alpha$-Klotho and PTH. Figure 6 ( $\alpha$-Klotho and PHOS) shows the meta-analysis of the correlation between $\alpha$-Klotho and PHOS. We included 5 studies, 3 of them found a positive correlation between $\alpha$-Klotho and PHOS, and 2 reported a negative correlation. With a random effects model $\left(\mathrm{I}^{2}=46.0 \%\right.$, $\mathrm{P}=0.116)$, the pooled Spearman correlation coefficient was 0.05 (95\%CI, -0.15 0.05, P>0.05), suggesting no significant correlation between $\alpha$-Klotho and PHOS. Egger's test or with Begg's test show no publication bias. The distribution of funnel plots was symmetrical (Supplementary Materials Figures 5,7, and 9). The result is suggestive of an indistinctive small study bias. In the sensitivity analysis, the pooled RR ranged from 0.05 (95\%CI, $-0.03 \sim 0.13)$ to 0.16 (95\%CI, 0.04 0.28) (Supplementary Materials Figure 6) of $\mathrm{Ca}$, the pooled RR ranged from -0.22 (95\%CI, $-0.33 \sim-0.12)$ to -0.07 (95\%CI, -0.30 0.17) (Supplementary Materials Figure 8) of PTH, and the pooled RR ranged from -0.08 (95\%CI, $-0.19 \sim 0.08)$ to 0.00 (95\%CI, -0.09 0.08) (Supplementary Materials Figure 10) of PHOS. No study had a significant impact on the total combined results.

\section{Discussion}

Kidney is a major organ to maintain soluble Klotho homeostasis by two ways. One is to cleavage membrane-bound 


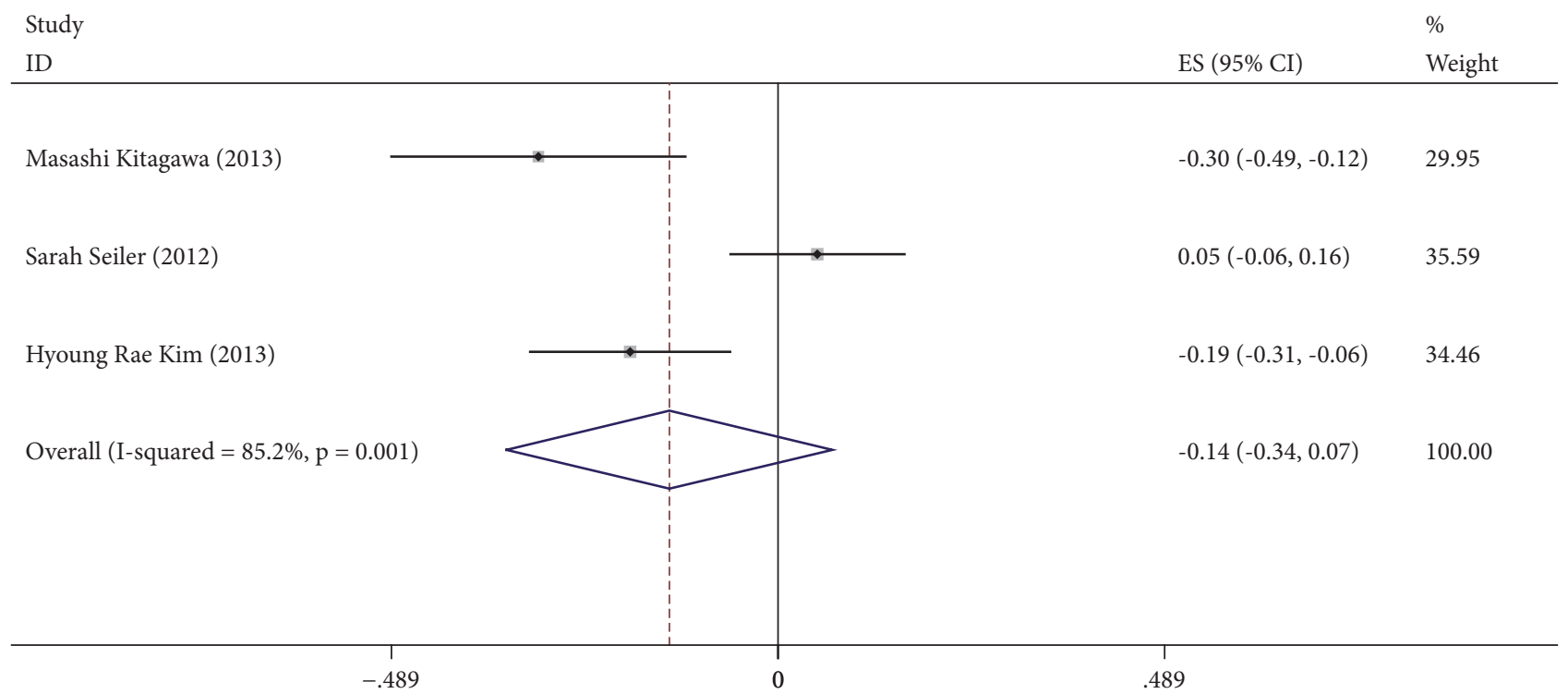

FIGURE 5: Forest plots of the summary correlation coefficient (r) with corresponding 95\% CIs for the correlation between $\alpha$-Klotho and PTH in patients from all eligible studies.

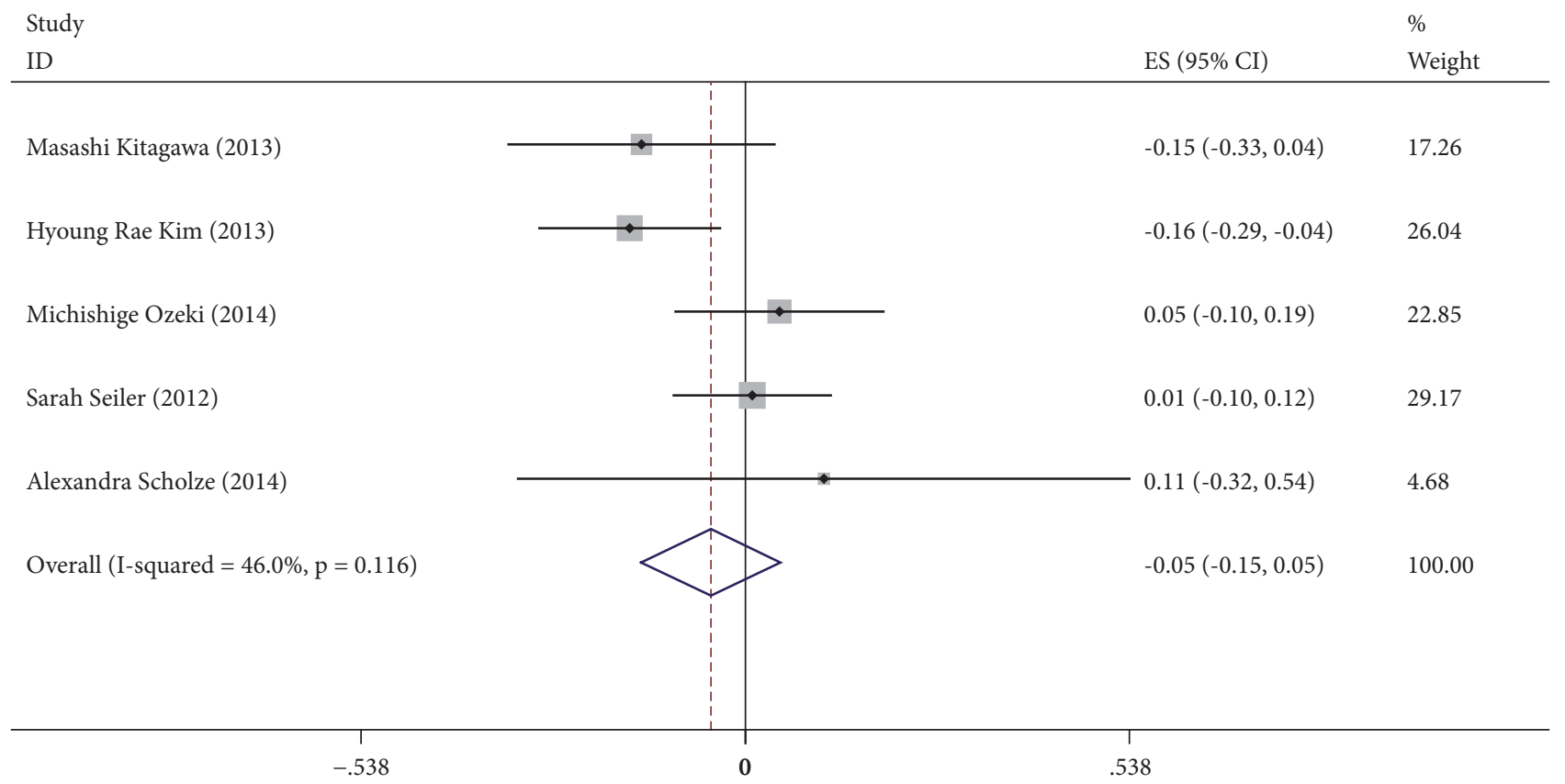

FIGURE 6: Forest plots of the summary correlation coefficient ( $\mathrm{r}$ ) with corresponding 95\% CIs for the correlation between $\alpha$-Klotho and PHOS in patients from all eligible studies.

$\alpha$-Klotho in the renal tubular epithelial cells and release into circulation. And the second is to eliminate redundant and unnecessary soluble $\alpha$-Klotho from circulations into the urinary lumen through renal proximal tubules by transcytosis to play its biological role [30]. In recent years, the correlation between soluble $\alpha$-Klotho and renal function in patients with CKD has been intensively studied [14, 15, 17, 18, 31]. A group of studies showed that there existed a sustained $\alpha$-Klotho deficiency status in patients with CKD and soluble $\alpha$-Klotho levels gradually became lower as eGFR declined [32-34]. In elderly well functioning adults, there also exists a longitudinal association between low soluble Klotho and decline in kidney function by a large, diverse cohort [35]. These findings raise hope to employ $\alpha$-Klotho as an early biomarker for renal function decline in patients with CKD. However, contrary to what was mentioned above, some studies found that soluble $\alpha$-Klotho levels had no significant correlation with eGFR [1719] or even two studies reported $\alpha$-Klotho were higher in 
patients with CKD $[31,36]$, we performed this review and meta-analysis to assess the correlation between soluble $\alpha$ Klotho levels and renal function in patients with CKD.

In this meta-analysis, with a random effects model, we found a significant positive correlation between soluble $\alpha$ Klotho and eGFR in patients with CKD as the pooled Spearman correlation coefficient was 0.35 (95\%CI, 0.23 $0.46)$. And the subgroup analysis revealed that there was no significant heterogeneity between subgroups including age, study area, and eGFR, which suggests that soluble $\alpha$ Klotho level declines as renal function decreases independent of age, study area, and eGFR. This conclusion was in accordance with previous studies [14-16]. As $\alpha$-Klotho is expressed in multiple tissues, the strongest expression by far is in kidney and also experimental data has showed that kidney directly contributes to circulating $\alpha$-Klotho [30]. It can be surmised that if kidney injuries, fibrosis occurs and renal function declines, its production would decrease (including membrane $\alpha$-Klotho and soluble $\alpha$-Klotho) [15], which has been proved by a prior study that the level of mRNA expression and protein production of $\alpha$-Klotho both reduced significantly in the kidneys among patients with chronic renal failure [34]. And a recent study demonstrated the serum soluble $\alpha$-Klotho levels significantly decreased after retroperitoneoscopic nephrectomy in 2 days and 5 days in living donors, respectively [37]. Furthermore, as for CKD patients, uremic toxins like indoxyl sulfate or p-cresyl sulfate induce hypermethylation of CpG dinucleotides in the $\alpha$ Klotho gene and inhibit $\alpha$-Klotho expression [38, 39]. These findings suggested that $\alpha$-Klotho has obvious correlation with renal function in multiple studies.

As a humoral regulator, $\alpha$-Klotho exerts many effects including antiaging, antioxidative stress, and antiapoptosis $[40,41]$. In experimental models, there has been ample evidence proving that $\alpha$-Klotho has a protective effect against kidney injury by gene transfer. Sugiura et al. found that Ad-kl (adenovirus harbouring the mouse Klotho gene) gene transfer ameliorated apoptosis attenuated tubular damage and reduced serum creatinine in ischaemic acute renal injury [42]. And Mitani et al. proved that Ad-kl gene transfer can ameliorate morphological kidney damage and improve creatinine clearance in angiotensin II-induced renal damage [43]. Similar findings were also seen in an immune-complex glomerulonephritis model [44]. Hence, it could be speculated that as kidney function declines, the production of $\alpha$ Klotho decreases and, due to lack of the protective effects of $\alpha$-Klotho, the kidney function deteriorates further, thus forming a vicious cycle. So it can be concluded that $\alpha$-Klotho might act as a prospective factor for renal function decline and replacement therapy may act as a crucial method. But large prospective follow-up cohorts are expected to conduct to validate our conclusions.

Conceivably, restoring $\alpha$-Klotho levels by reactivating endogenous expression of $\alpha$-Klotho gene or providing exogenous $\alpha$-Klotho might be a novel therapeutic target for patients with CKD [45]. And some rodent animal experiments have already proved that bolus supplementation of soluble Klotho protein is safe and effective for protecting kidney function and retarding CKD progression [46-48]. Experiments on primates or even clinical experiments are in urgent needs.

In our meta-analysis, we also found a significant negative correlation between $\alpha$-Klotho and FGF23 levels in CKD patients. With a random effects model $\left(\mathrm{I}^{2}=56.6 \%\right.$, $\mathrm{P}=0.032)$, the pooled Spearman correlation coefficient was 0.10 (95\%CI, $-0.19 \sim-0.01$, and $\mathrm{P}<0.05)$. The same conclusions were also made by other studies [16, 49-51]. On the one hand, as soluble $\alpha$-Klotho is mainly generated by proteolytic release of the transmembrane form in kidney, soluble $\alpha$-Klotho is a reflector of renal $\alpha$-Klotho expression [8] and the two forms of $\alpha$-Klotho would decrease together when the renal function declines. As a coreceptor for FGF23, a decreased expression of transmembrane $\alpha$-Klotho would contribute to a compensatory increase in FGF23 to maintain mineral homeostasis [19]. So in early stage of CKD, serum phosphate is not elevated. With kidney function deteriorating, $\alpha$-Klotho further decreases and exacerbates FGF23 resistance. And the elevated FGF23 in turn decreases $\alpha$-Klotho expression [52], thus forming a vicious cycle. Finally the elevated FGF23 levels can no longer compensate for dietary phosphorus overload which contributes to overt hyperphosphatemia in advanced stages of CKD. On the other hand, soluble $\alpha$-Klotho can inhibit sodium phosphate cotransporters in the proximal tubules, thus promoting urinary phosphate excretion [10]. A decrease in soluble $\alpha$-Klotho may result an increase in serum phosphate levels due to increased phosphate absorption, which in turn can stimulate FGF-23 production. However, Isakova et al. [51] found there was a subtle decrease in serum phosphate in the early stages of CKD. Meanwhile, levels of FGF23 and fractional excretion of phosphate (FEPi) increased and 1,25D levels decreased. They inclined to that these syndromes were in accordance with primary FGF23 excess rather than primary $\alpha$-Klotho deficiency. Because if Klotho deficiency is the initial factor that causes secondary increases in FGF23 secretion, the subtle signs of Klotho deficiency would be manifested in the early stage of CKD, such as increases in serum phosphate, 1,25D and calcium levels. However, the observed syndromes were contrary to what was mentioned above. And there is an alternative, unifying explanation that increases in FGF23 is the initial factor causing secondary downregulating of $\alpha$ Klotho by hypophosphatemia or reduced 1,25D3 [53]. And Tamara Isakova et al. [51] supposed that maybe by increasing expression of a stimulus for FGF23 secretion or decreasing production of an inhibitor, the kidney injury itself is an initial stimulus for FGF23 increasing in early CKD. Its possibility is supported by the animal studies: when kidney was injured, FGF23 rose immediately, which precedes any increase in serum phosphate [54]. To date, it is still unclear which one of FGF-23 upregulation and $\alpha$-Klotho deficiency occurs first.

By the subgroup analysis, we found that the correlation varied as study area differed; for example, participants in Asia subgroup demonstrated a significant negative correlation between $\alpha$-Klotho and FGF23, while participants in Europe subgroup demonstrated no statistical correlations. The following ideas may, to some extent, explain the differences: firstly, the genetic differences exist among different races, which may influence the expression of soluble $\alpha$-Klotho and 
FGF23; secondly, there is only 3 publications included in Europe subgroup, and maybe we can get significant result by increasing the sample size; thirdly, as mentioned above, the levels of serum phosphate are a stimulus for FGF23. And the serum phosphate levels among different countries may be variable due to dietary phosphorus intake, medication, socioeconomic status, and other factors. For example, it has been reported that there is an independent association between poverty and higher serum phosphate levels $[55,56]$. We supposed that participants in Europe may have a better control of serum phosphorus and that the level of FGF23 is relatively lower, which needs to be validated by further clinical study.

In this analysis, we also figured out the correlations between soluble $\alpha$-Klotho and serum phosphorus, PTH, and calcium respectively, but failed to confirm its correlations statistically. As an autocrine or paracrine enzyme, soluble $\alpha$ Klotho reduces urine phosphorus reabsorption by inhibiting the expression of renal sodium phosphate cotransporter $\mathrm{NaPi}-2 \mathrm{a}[10]$ and increases urine calcium uptake by stabilizing TRPV5 calcium channel [57] independent of FGF23. It is reported that parathyroid resistance, an increase of FGF23 failing to decrease parathyroid hormone levels, is mediated, in part, by depressed expression of $\alpha$-Klotho [58-60]. It can be speculated that soluble $\alpha$-Klotho can decrease serum phosphate and PTH levels as well as elevate serum calcium levels. But it has been reported that there was a significant inverse correlation between serum Ca and soluble $\alpha$-Klotho in Children with CKD [61]. And among most patients with mild-to-moderate CKD, serum phosphate levels are normal due to compensatory increases in FGF23 and PTH $[49,62]$. And $\alpha$-Klotho is only one of the factors regulating mineral metabolism. The levels of serum phosphorus, PTH, and calcium are influenced by so many other factors such as transmembrane $\alpha$-Klotho, soluble $\alpha$-Klotho, FGF23, 1,25D3, dietary, and medication and these factors are interacting with each other by complicated ways. We cannot completely eliminate the effects of these factors on our analysis.

In summary, as kidney function declines, soluble $\alpha$ Klotho decreases and FGF23 increases. But it is still unclear which one of them occurs first. At its early stages, these changes could help maintain mineral homeostasis. With kidney function deteriorating, the suppressive effect of increased FGF23 on serum phosphate and PTH production is defunctionalized due to furtherly decreased $\alpha$-Klotho. As we could see, hyperphosphatemia and secondary hyperparathyroidism (SHPT) occur. And 1,25D3 decreases when kidney function declines, due to it is produced in kidney. Also, it can be suppressed by increased FGF23, which leads to decreased serum calcium. Low calcium and hyperphosphatemia further exacerbate SHPT and elevate PTH and FGF23 levels. Changes above form a vicious cycle and in concert contribute to the development of complications of CKD, such as metabolic bone disease, SHPT and vascular calcification (Figure 7).

Limitations. Our study still has some inherent limitations. Firstly, although the total number of involved patients in our meta-analysis is relative large, the number of the participants in some of the included studies is quite small. In this case, the

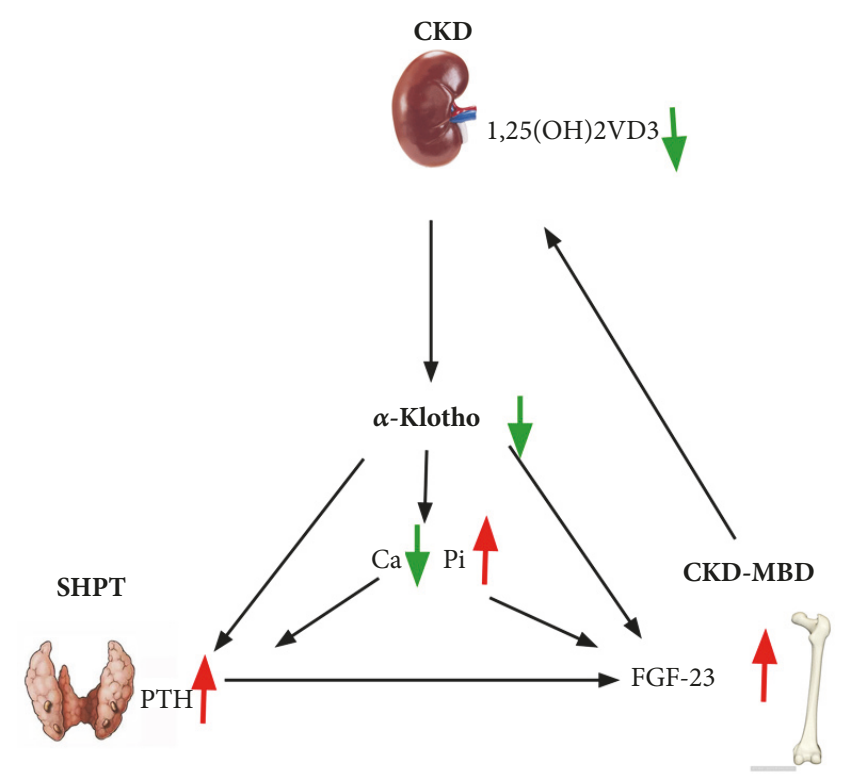

FIgURE 7: Cross connection diagram of hypothesis.

conclusions in them would be less convincing. Secondly, our analysis is based on studies that had been published, which tends to reveal positive and significant results. Some studies with negative and insignificant results might be rejected. Moreover, some results associated with our analysis are still on the way to be accepted. This would cause publications bias. Thirdly, in some clinical researches and basic researches, they revealed positive or negative correlation between these indexes, but no $\mathrm{R}$ values or raw data were supplemented to calculate correlation coefficient. Fourthly, our meta-analysis is restricted to articles published in English, which would lead to publications bias. However, the results of Begg's test showed no evidence of publication bias. We also used the random effects model to reduce heterogeneity. Therefore, the results of the present study are reliable.

In conclusion, although certain limitations still exist in this meta-analysis, the conclusions we obtained currently support that there exists a significant positive correlation between soluble $\alpha$-Klotho and eGFR in patients with CKD. Also, a significant negative correlation between $\alpha$-Klotho and FGF23 levels is found. This raises hope to employ $\alpha$-Klotho and FGF23 as candidates of biomarkers of CKD patients with high sensitivity and specificity, preceding decline dramatically with different CKD stages and disturbance of CKDmineral metabolism. However a large prospective cohort study and basic research are still in urgent needs to consolidate our conclusions and verify the following: (a) a detailed mechanism that kidney how to maintain $\alpha$-Klotho homeostasis; (b) FGF-23 upregulation, $\alpha$-Klotho deficiency, which occurs first; (c) whether replacement therapy (exogenous and endogenous) is safe and clinically effective.

\section{Disclosure}

Qinglian Wang and Wenyan Su are co-first authors. 


\section{Conflicts of Interest}

All authors declare that they have no conflicts of interest.

\section{Authors' Contributions}

Rong Wang conceived and designed the meta-analysis; Qinglian Wang and Wenyan Su searched literatures and extracted data; Qinglian Wang and Zhenwei Shen analyzed the data. All authors reviewed the manuscript and approved the submitted version.

\section{Supplementary Materials}

Supplementary Materials Figure 1. To detect the study bias, we conducted funnel plot of the publication bias for $\alpha$-Klotho and eGFR group. The result is suggestive of an indistinctive small study bias. Supplementary Materials Figure 2. To test whether a study has a significant impact on the results, sensitivity estimates for $\alpha$-Klotho and eGFR group with corresponding 95\% CIs were conducted. In the sensitivity analysis, the pooled RR ranged from 0.32 (95\%CI, 0.19 0.44) to 0.37 (95\%CI, 0.26 0.48). No study had a significant impact on the total combined results. Supplementary Materials Figure 3. To detect the study bias, we conducted funnel plot of the publication bias for $\alpha$-Klotho and FGF-23 group. The result is suggestive of an indistinctive small study bias. Supplementary Materials Figure 4. To test whether a study has a significant impact on the results, sensitivity estimates for $\alpha$-Klotho and FGF-23 group with corresponding 95\% CIs were conducted. In the sensitivity analysis, the pooled RR ranged from 0.13 (95\%CI, -0.20 -0.06) to -0.07(95\%CI, -0.17 -0.02). No study had a significant impact on the total combined results. Supplementary Materials Figure 5. To detect the study bias, we conducted funnel plot of the publication bias for $\alpha$-Klotho and Ca group. The result is suggestive of an indistinctive small study bias. Supplementary Materials Figure 6. To test whether a study has a significant impact on the results, sensitivity estimates for $\alpha$-Klotho and Ca group with corresponding $95 \%$ CIs were conducted. In the sensitivity analysis, the pooled RR ranged from 0.05 (95\%CI, $-0.03 \sim 0.13)$ to 0.16 (95\%CI, 0.04 0.28). No study had a significant impact on the total combined results. Supplementary Materials Figure 7. To detect the study bias, we conducted funnel plot of the publication bias for $\alpha$-Klotho and PTH group. The result is suggestive of an indistinctive small study bias. Supplementary Materials Figure 8. To test whether a study has a significant impact on the results, sensitivity estimates for $\alpha$-Klotho and PTH group with corresponding 95\% CIs was conducted. In the sensitivity analysis, the pooled RR ranged from 0.22 (95\%CI, -0.33 -0.12) to -0.07 (95\%CI, $-0.30 \sim 0.17$ ). No study had a significant impact on the total combined results. Supplementary Materials Figure 9. To detect the study bias, we conducted funnel plot of the publication bias for $\alpha$-Klotho and PHOS group. The result is suggestive of an indistinctive small study bias. Supplementary Materials Figure 10. To test whether a study has a significant impact on the results, sensitivity estimates fo $\alpha$-Klotho and PHOS group with corresponding 95\% CIs were conducted. In the sensitivity analysis, the pooled RR ranged from -0.08 (95\%CI, -0.19 0.08) to 0.00 (95\%CI, -0.09 0.08). No study had a significant impact on the total combined results. (Supplementary Materials)

\section{References}

[1] M. Kuro-o, Y. Matsumura, H. Aizawa et al., "Mutation of the mouse klotho gene leads to a syndrome resembling ageing," Nature, vol. 390, no. 6655, pp. 45-51, 1997.

[2] I. Z. Ben-Dov, H. Galitzer, V. Lavi-Moshayoff et al., "The parathyroid is a target organ for FGF23 in rats," The Journal of Clinical Investigation, vol. 117, no. 12, pp. 4003-4008, 2007.

[3] M. C. Neidert, L. Sze, C. Zwimpfer et al., "Soluble -Klotho: a novel serum biomarker for the activity of GH-producing pituitary adenomas," European Journal of Endocrinology, vol. 168, no. 4, pp. 575-583, 2013.

[4] M. Kamemori, Y. Ohyama, M. Kurabayashi, K. Takahashi, R. Nagai, and N. Furuya, "Expression of Klotho protein in the inner ear," Hearing Research, vol. 171, no. 1-2, pp. 103-110, 2002.

[5] I. Wolf, S. Levanon-Cohen, S. Bose et al., "Klotho: a tumor suppressor and a modulator of the IGF-1 and FGF pathways in human breast cancer," Oncogene, vol. 27, no. 56, pp. 7094-7105, 2008.

[6] J. Bernheim and S. Benchetrit, "The potential roles of FGF23 and Klotho in the prognosis of renal and cardiovascular diseases," Nephrology Dialysis Transplantation, vol. 26, no. 8, pp. $2433-$ 2438, 2011.

[7] Y. Wang and Z. Sun, "Current understanding of klotho," Ageing Research Reviews, vol. 8, no. 1, pp. 43-51, 2009.

[8] T. E. Larsson, "The role of FGF-23 in CKD-MBD and cardiovascular disease: friend or foe?” Nephrology Dialysis Transplantation, vol. 25, no. 5, pp. 1376-1381, 2010.

[9] A. Imura, A. Iwano, O. Tohyama et al., "Secreted Klotho protein in sera and CSF: implication for post-translational cleavage in release of Klotho protein from cell membrane," FEBS Letters, vol. 565, no. 1-3, pp. 143-147, 2004.

[10] M. C. Hu, M. Shi, J. Zhang et al., "Klotho: A novel phosphaturic substance acting as an autocrine enzyme in the renal proximal tubule," The FASEB Journal, vol. 24, no. 9, pp. 3438-3450, 2010.

[11] M. Kuro-o, "Klotho and aging," Biochimica et Biophysica Acta (BBA) - General Subjects, vol. 1790, no. 10, pp. 1049-1058, 2009.

[12] V. Jha, G. Garcia-Garcia, K. Iseki et al., "Chronic kidney disease: global dimension and perspectives," The Lancet, vol. 382, no. 9888, pp. 260-272, 2013.

[13] G. Manjunath, H. Tighiouart, H. Ibrahim et al., "Level of kidney function as a risk factor for atherosclerotic cardiovascular outcomes in the community," Journal of the American College of Cardiology, vol. 41, no. 1, pp. 47-55, 2003.

[14] M. Kitagawa, H. Sugiyama, H. Morinaga et al., "A decreased level of serum soluble Klotho is an independent biomarker associated with arterial stiffness in patients with chronic kidney disease," PLoS ONE, vol. 8, no. 2, Article ID e56695, 2013.

[15] H. R. Kim, B. Y. Nam, D. W. Kim et al., "Circulating alphaklotho levels in CKD and relationship to progression," American Journal of Kidney Diseases, vol. 61, no. 6, pp. 899-909, 2013.

[16] Y. Shimamura, K. Hamada, K. Inoue et al., "Serum levels of soluble secreted a-Klotho are decreased in the early stages of chronic kidney disease, making it a probable novel biomarker for early diagnosis," Clinical and Experimental Nephrology, vol. 16, no. 5, pp. 722-729, 2012. 
[17] T. Akimoto, H. Yoshizawa, Y. Watanabe et al., "Characteristics of urinary and serum soluble Klotho protein in patients with different degrees of chronic kidney disease," BMC Nephrology, vol. 13, article 155, 2012.

[18] V. Hage, S. Pelletier, L. Dubourg et al., "In chronic kidney disease, serum $\alpha$-Klotho is related to serum bicarbonate and proteinuria," Journal of Renal Nutrition, vol. 24, no. 6, pp. 390394, 2014

[19] S. Seiler, M. Wen, H. J. Roth et al., "Plasma Klotho is not related to kidney function and does not predict adverse outcome in patients with chronic kidney disease," Kidney International, vol. 83, no. 1, pp. 121-128, 2013.

[20] E. von Elm, D. G. Altman, M. Egger, S. J. Pocock, P. C. Gøtzsche, and J. P. Vandenbroucke, "The strengthening the reporting of observational studies in epidemiology (STROBE) statement: guidelines for reporting observational studies," International Journal of Surgery, vol. 12, no. 12, pp. 1495-1499, 2014.

[21] P. Jüni, A. Witschi, R. Bloch, and M. Egger, "The hazards of scoring the quality of clinical trials for meta-analysis," The Journal of the American Medical Association, vol. 282, no. 11, pp. 1054-1060, 1999.

[22] A. Stang, "Critical evaluation of the Newcastle-Ottawa scale for the assessment of the quality of nonrandomized studies in metaanalyses," European Journal of Epidemiology, vol. 25, no. 9, pp. 603-605, 2010.

[23] J. M. Hootman, J. B. Driban, M. R. Sitler, K. P. Harris, and N. M. Cattano, "Reliability and validity of three quality rating instruments for systematic reviews of observational studies," Research Synthesis Methods, vol. 2, no. 2, pp. 110-118, 2011.

[24] L. Chen, M. Liu, J. Bao et al., "The correlation between apparent diffusion coefficient and tumor cellularity in patients: a metaanalysis," PLoS ONE, vol. 8, no. 11, Article ID e79008, 2013.

[25] D. B. Wilson and M. W. Lipsey, "The role of method in treatment effectiveness research: Evidence from meta-analysis," Psychological Methods, vol. 6, no. 3, pp. 413-429, 2001.

[26] T. A. McGrath, M. Alabousi, B. Skidmore et al., "Recommendations for reporting of systematic reviews and meta-analyses of diagnostic test accuracy: a systematic review," Systematic Reviews, vol. 6, no. 1, 2017.

[27] M. Ozeki, S.-I. Fujita, S. Kizawa et al., "Association of serum levels of FGF23 and $\alpha$-Klotho with glomerular filtration rate and proteinuria among cardiac patients," BMC Nephrology, vol. 15, no. 1, p. 147, 2014.

[28] I. Pavik, P. Jaeger, L. Ebner et al., "Secreted Klotho and FGF23 in chronic kidney disease stage 1 to 5 : a sequence suggested from a cross-sectional study," Nephrology Dialysis Transplantation, vol. 28, no. 2, pp. 352-359, 2013.

[29] A. Scholze, Y. Liu, L. Pedersen et al., "Soluble a-Klotho and Its Relation to Kidney Function and Fibroblast Growth Factor-23," The Journal of Clinical Endocrinology \& Metabolism, vol. 99, no. 5, pp. E855-E861, 2014.

[30] M. C. Hu, M. Shi, J. Zhang et al., "Renal production, uptake, and handling of circulating $\alpha$ Klotho," Journal of the American Society of Nephrology, vol. 27, no. 1, pp. 79-90, 2015.

[31] H. Sugiura, K. Tsuchiya, and K. Nitta, "Circulating levels of soluble $\alpha$-Klotho in patients with chronic kidney disease," Clinical and Experimental Nephrology, vol. 15, no. 5, pp. 795-796, 2011.

[32] O. Asai, K. Nakatani, T. Tanaka et al., "Decreased renal $\alpha$ Klotho expression in early diabetic nephropathy in humans and mice and its possible role in urinary calcium excretion," Kidney International, vol. 81, no. 6, pp. 539-547, 2012.
[33] H. Sakan, K. Nakatani, and O. Asai, "Reduced renal $\alpha$-Klotho expression in CKD patients and its effect on renal phosphate handling and vitamin D metabolism," PLOS ONE, vol. 9, no. 1, Article ID e86301, 2014.

[34] N. Koh, T. Fujimori, S. Nishiguchi et al., "Severely reduced production of klotho in human chronic renal failure kidney," Biochemical and Biophysical Research Communications, vol. 280, no. 4, pp. 1015-1020, 2001.

[35] D. A. Drew, R. Katz, S. Kritchevsky et al., "Association between soluble klotho and change in kidney function: The health aging and body composition study," Journal of the American Society of Nephrology, vol. 28, no. 6, pp. 1859-1866, 2017.

[36] S. Devaraj, B. Syed, A. Chien, and I. Jialal, "Validation of an immunoassay for soluble Klotho protein: decreased levels in diabetes and increased levels in chronic kidney disease," American Journal of Clinical Pathology, vol. 137, no. 3, pp. 479-485, 2012.

[37] T. Akimoto, T. Kimura, and Y. Watanabe, "The impact of nephrectomy and renal transplantation on serum levels of soluble Klotho protein," Transplantation Proceedings, vol. 45, no. 1, pp. 134-136, 2013.

[38] J. Chen, X. Zhang, H. Zhang et al., "Elevated Klotho Promoter Methylation Is Associated with Severity of Chronic Kidney Disease," PLoS ONE, vol. 8, no. 11, p. e79856, 2013.

[39] G.-H. Young and V.-C. Wu, "KLOTHO methylation is linked to uremic toxins and chronic kidney disease," Kidney International, vol. 81, no. 7, pp. 611-612, 2012.

[40] H. Kurosu, M. Yamamoto, J. D. Clark et al., "Physiology: suppression of aging in mice by the hormone Klotho," Science, vol. 309, no. 5742, pp. 1829-1833, 2005.

[41] M. Kuro-o, "Klotho as a regulator of oxidative stress and senescence," biological chemistry, vol. 389, no. 3, pp. 233-241, 2008.

[42] H. Sugiura, T. Yoshida, K. Tsuchiya et al., "Klotho reduces apoptosis in experimental ischaemic acute renal failure," Nephrology Dialysis Transplantation, vol. 20, no. 12, pp. 2636-2645, 2005.

[43] H. Mitani, N. Ishizaka, T. Aizawa et al., "In vivo klotho gene transfer ameliorates angiotensin II-induced renal damage," Hypertension, vol. 39, no. 4, pp. 838-843, 2002.

[44] Y. Haruna, N. Kashihara, M. Satoh et al., "Amelioration of progressive renal injury by genetic manipulation of Klotho gene," Proceedings of the National Acadamy of Sciences of the United States of America, vol. 104, no. 7, pp. 2331-2336, 2007.

[45] X. Lu and M. C. Hu, "Klotho/FGF23 Axis in Chronic Kidney Disease and Cardiovascular Disease," Kidney Diseases, vol. 3, no. 1, pp. 15-23, 2017.

[46] M.-C. Hu, M. Shi, J. Zhang, H. Quĩones, M. Kuro-O, and O. W. Moe, "Klotho deficiency is an early biomarker of renal ischemia-reperfusion injury and its replacement is protective," Kidney International, vol. 78, no. 12, pp. 1240-1251, 2010.

[47] M. Shi, B. Flores, N. Gillings et al., " $\alpha$ klotho mitigates progression of aki to ckd through activation of autophagy," Journal of the American Society of Nephrology, vol. 27, no. 8, pp. 2331-2345, 2016.

[48] S. Doi, Y. Zou, O. Togao et al., "Klotho inhibits transforming growth factor- $\beta 1$ (TGF- $\beta 1$ ) signaling and suppresses renal fibrosis and cancer metastasis in mice," The Journal of Biological Chemistry, vol. 286, no. 10, pp. 8655-8665, 2011.

[49] O. Gutierrez, T. Isakova, E. Rhee et al., "Fibroblast growth factor-23 mitigates hyperphosphatemia but accentuates calcitriol deficiency in chronic kidney disease," Journal of the 
American Society of Nephrology, vol. 16, no. 7, pp. 2205-2215, 2005.

[50] T. Shigematsu, J. J. Kazama, T. Yamashita et al., "Possible involvement of circulating fibroblast growth factor 23 in the development of secondary hyperparathyroidism associated with renal insufficiency," American Journal of Kidney Diseases, vol. 44, no. 2, pp. 250-256, 2004.

[51] T. Isakova, P. Wahl, G. S. Vargas et al., "Fibroblast growth factor 23 is elevated before parathyroid hormone and phosphate in chronic kidney disease," Kidney International, vol. 79, no. 12, pp. 1370-1378, 2011.

[52] R. Marsell, T. Krajisnik, H. Göransson et al., "Gene expression analysis of kidneys from transgenic mice expressing fibroblast growth factor-23," Nephrology Dialysis Transplantation, vol. 23, no. 3, pp. 827-833, 2008.

[53] H. Tsujikawa, Y. Kurotaki, T. Fujimori, K. Fukuda, and Y.-I. Nabeshima, "Klotho, a gene related to a syndrome resembling human premature aging, functions in a negative regulatory circuit of vitamin D endocrine system," Molecular Endocrinology, vol. 17, no. 12, pp. 2393-2403, 2003.

[54] H. Hasegawa, N. Nagano, I. Urakawa et al., "Direct evidence for a causative role of FGF23 in the abnormal renal phosphate handling and vitamin D metabolism in rats with early-stage chronic kidney disease," Kidney International, vol. 78, no. 10, pp. 975-980, 2010.

[55] O. M. Gutiérrez, T. Isakova, G. Enfield, and M. Wolf, "Impact of Poverty on Serum Phosphate Concentrations in the Third National Health and Nutrition Examination Survey," Journal of Renal Nutrition, vol. 21, no. 2, pp. 140-148, 2011.

[56] O. M. Gutiérrez, C. Anderson, T. Isakova et al., "Low socioeconomic status associates with higher serum phosphate irrespective of race," Journal of the American Society of Nephrology, vol. 21, no. 11, pp. 1953-1960, 2010.

[57] S.-K. Cha, B. Ortega, H. Kurosu, K. P. Rosenblatt, M. Kuro, and C.-L. Huang, "Removal of sialic acid involving Klotho causes cell-surface retention of TRPV5 channel via binding to galectin1," Proceedings of the National Acadamy of Sciences of the United States of America, vol. 105, no. 28, pp. 9805-9810, 2008.

[58] H. Galitzer, I. Z. Ben-Dov, J. Silver, and T. Naveh-Many, "Parathyroid cell resistance to fibroblast growth factor 23 in secondary hyperparathyroidism of chronic kidney disease," Kidney International, vol. 77, no. 3, pp. 211-218, 2010.

[59] H. Komaba, S. Goto, H. Fujii et al., "Depressed expression of Klotho and FGF receptor 1 in hyperplastic parathyroid glands from uremic patients," Kidney International, vol. 77, no. 3, pp. 232-238, 2010.

[60] R. Canalejo, A. Canalejo, J. M. Martinez-Moreno et al., "FGF23 fails to inhibit uremic parathyroid glands," Journal of the American Society of Nephrology, vol. 21, no. 7, pp. 1125-1135, 2010.

[61] H. K. Sawires, R. M. Essam, M. F. Morgan, and R. A. Mahmoud, "Serum klotho: relation to fibroblast growth factor-23 and other regulators of phosphate metabolism in children with chronic kidney disease," Nephron, vol. 129, no. 4, pp. 293-299, 2015.

[62] A. Levin, G. L. Bakris, M. Molitch et al., "Prevalence of abnormal serum vitamin $\mathrm{D}, \mathrm{PTH}$, calcium, and phosphorus in patients with chronic kidney disease: results of the study to evaluate early kidney disease," Kidney International, vol. 71, no. 1, pp. 31-38, 2007. 


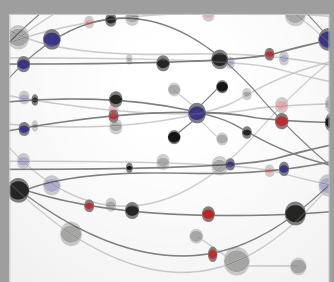

The Scientific World Journal
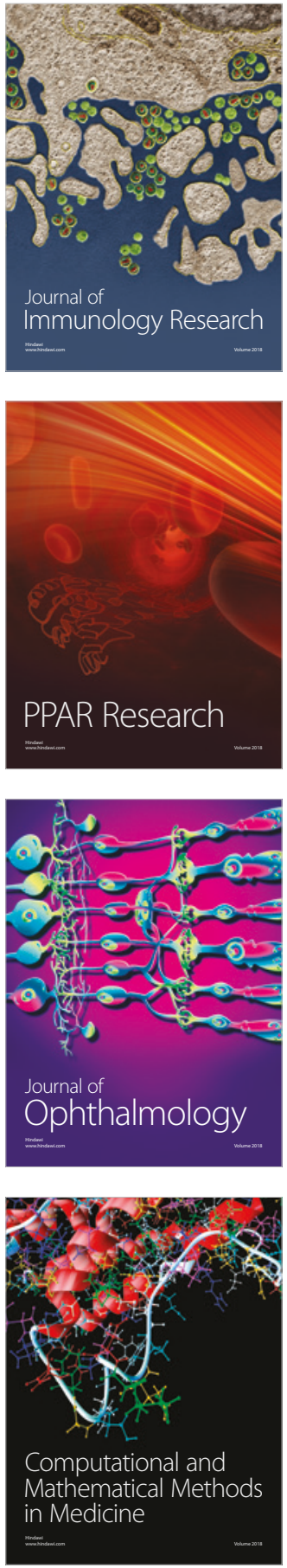

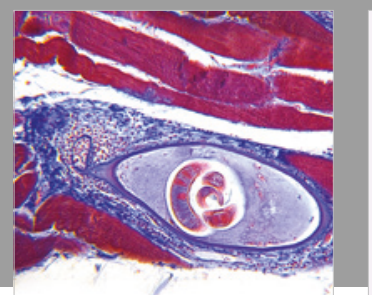

Gastroenterology Research and Practice

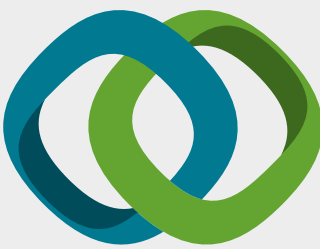

\section{Hindawi}

Submit your manuscripts at

www.hindawi.com
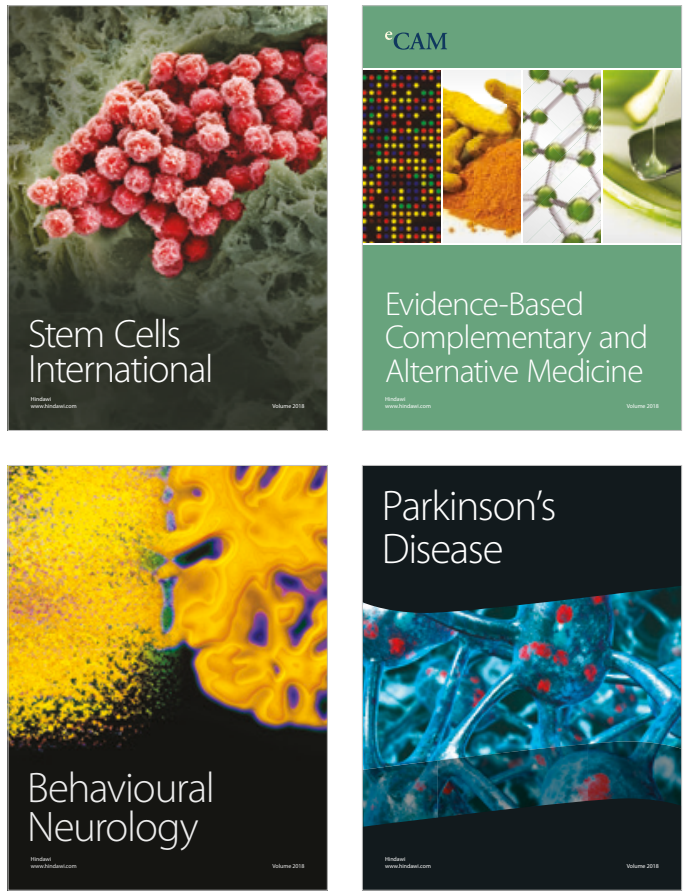

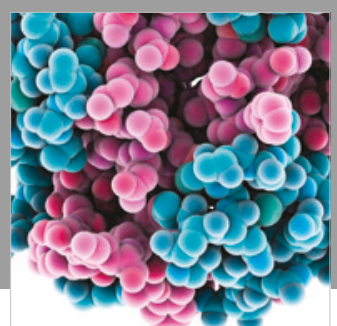

ournal of

Diabetes Research

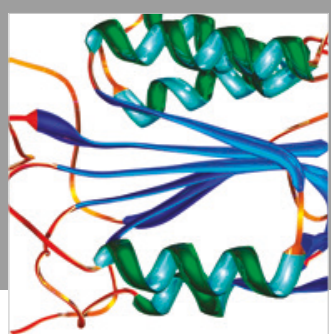

Disease Markers
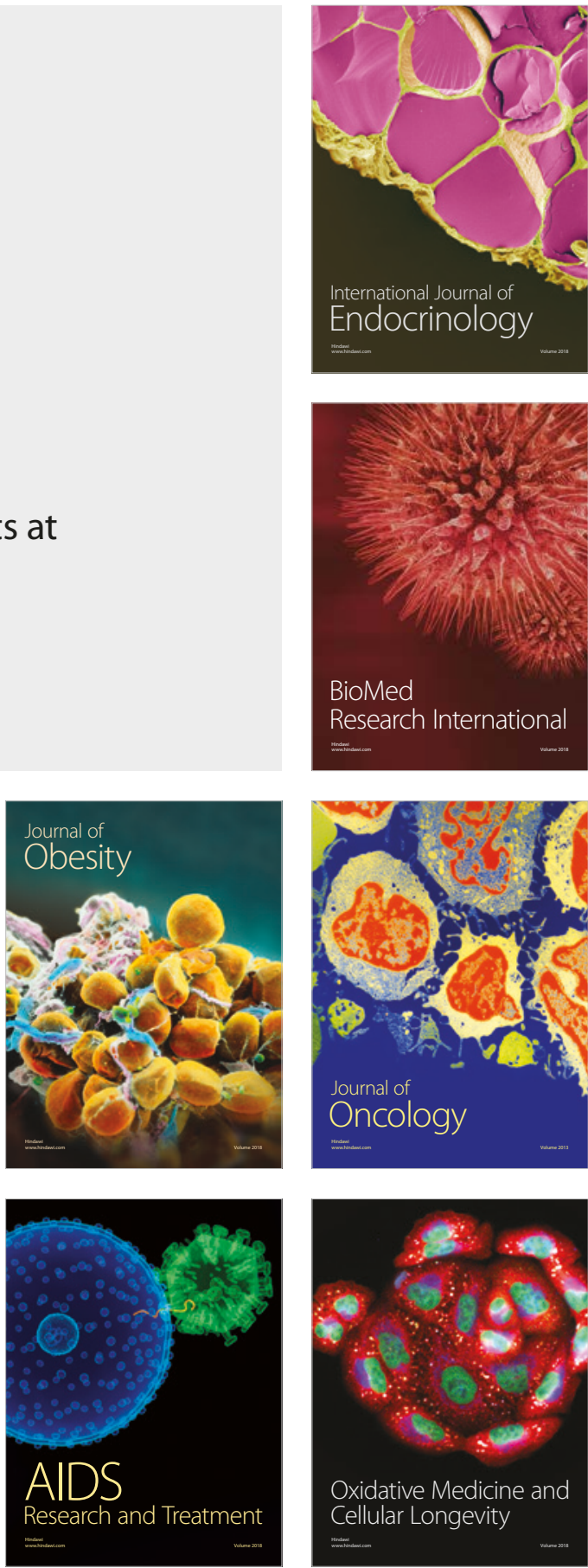